\section{DIMENSIONES Y DESAFÍOS DE LA PARTICIPACIÓN CIUDADANA EN LA GESTIÓN DE RIESGO DE DESASTRES EN ASUNCIÓN, ÁREA METROPOLITANA Y BAJO CHACO, PARAGUAY}

Marta Isabel Canese de Estigarribia ${ }^{1 *}$, Cecilia María Vuyk Espínola ${ }^{1}$, Romilio González Chamorro ${ }^{1}$, Alberto Aquiles Britez Acuña ${ }^{1}$, José Carlos Lezcano Villagra ${ }^{1}$ y Violeta Luciana Prieto Granada ${ }^{1}$

\section{RESUMEN}

Este estudio presenta una revisión de los antecedentes, dimensiones y desafíos de la participación ciudadana en la gestión de riesgo en la ciudad de Asunción, Paraguay, y municipios circundantes. La metodología aplicada fue la revisión bibliográfica sistematizada, desarrollada en sus cuatro fases: búsqueda, evaluación, análisis y síntesis. Los datos analizados fueron agrupados en cuatro dimensiones: histórica, sociodemográfica, ambiental y política. Los resultados obtenidos destacan la mención de la participación ciudadana como prioridad en los documentos referentes a políticas públicas internacionales y nacionales en gestión de riesgo. Sin embargo, esa participación se limita, en muchos casos, al aspecto formal, con escaso poder de la ciudadanía en la incidencia y el control de la gestión de riesgo a nivel nacional y local.

\section{PALABRAS CLAVES}

Gestión local, Políticas públicas, Ciudadanía, Sociedad civil, Paraguay

\section{DIMENSIONS AND CHALLENGES OF CITIZEN PARTICIPATION IN DISASTER RISK MANAGEMENT IN ASUNCIÓN, METROPOLITAN AREAAND SOUTH WESTERN REGION, PARAGUAY}

\section{ABSTRACT}

This study offers a review of the antecedents, dimensions, and challenges of citizen participation in risk management in the city of Asuncion in Paraguay and its surrounding municipalities. The applied methodology consisted of a systematized bibliographic review developed in four phases: search, evaluation, analysis, and synthesis. The analyzed data were grouped into four dimensions: historical, socio-demographic, environmental, and political. The obtained results highlight 'citizen participation' as a priority in documents referring to international and national public policies on risk management. In the selected case of study, however, this participation is mainly limited to only 'formal' aspects rather than observed in practice. The latter has left little power to citizens for the incidence and control of risk management at a national and local levels.

\section{KEYWORDS}

Local management, Public policies, Citizenship, Civil society, Paraguay

\author{
1. Escuela de Ciencias \\ Sociales y Políticas, \\ Universidad Nacional \\ de Asunción, Asunción, \\ Paraguay. \\ *Autor de correspondencia: \\ mcanese@gmail.com
}

\section{DOI:}

https://doi.org/10.55467/ reder.v6i1.87

\section{RECIBIDO}

4 de junio de 2021

\section{ACEPTADO}

12 de septiembre de 2021

\section{PUBLICADO}

1 de enero de 2022

\section{Formato cita}

Recomendada (APA):

Canese de Estigarribia,

M.I., Vuyk Espínola, C.M.,

González Chamorro,

R., Britez Acuña, A.A.,

Lezcano Villagra, J.C. \& Prieto Granada, V.L. (2022).

Dimensiones y desafíos de la participación ciudadana en la gestión de riesgo de desastres en Asunción, Área Metropolitana y Bajo Chaco, Paraguay. Revista de Estudios Latinoamericanos sobre Reducción del Riesgo de Desastres REDER, 6(1), 112123. https://doi.org/10.55467/ reder.v6i1.87

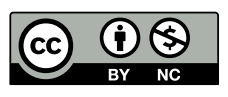

Todos los artículos publicados en REDER siguen una política de Acceso Abierto y se respaldan en una Licencia CreativeCommons Atribución-NoComercial 4.0 Internacional.

Revista de Estudios Latinoamericanos sobre Reducción del Riesgo de Desastres (REDER)

Diseño: Lupe Bezzina 


\section{INTRODUCCIÓN}

Este estudio tuvo por objetivo analizar las dimensiones sociodemográficas, históricas y políticas de la participación ciudadana para la gestión de riesgos por desastres en Asunción, Área Metropolitana y Bajo Chaco, en el periodo 2010-2020. El estudio adopta la orientación de la epistemología crítica, en la que el objeto de estudio no es una copia de la realidad, sino que propone la inclusión de dimensiones de la realidad en el objeto, reposicionando el problema de la neutralidad en la comprobación científica (Zemelman, 2011). El enfoque del estudio se centra en la construcción social de riesgo desde la participación ciudadana, donde las variables sociales, como participación ciudadana y vulnerabilidad, se integran con las ambientales. La investigación se fundamenta en la teoría crítica, estructurada en tres ejes teóricos que interactúan de forma dialéctica: la realidad social, el sujeto y el conocimiento (Zemelman, 2005). En este marco, la acción de los sujetos debe fortalecerse para construir un presente potencial de transformación social, sin imposiciones ni metas externas a su subjetividad. Para Zemelman (1989, p.68), el sujeto social "sintetiza en su experiencia una historicidad y un proyecto de futuro".

El concepto de desastres aplicado en esta investigación corresponde a una perspectiva crítica, y a la vez interdisciplinaria. Los desastres, en esta concepción, no son el resultado directo de un evento natural solamente. Su concepción implica un conjunto de factores interrelacionados, donde las desigualdades sociales, el comportamiento del ciudadano, la exclusión territorial, económica y social de colectivos vulnerables y las condiciones ambientales interactúan (Lavell, 2007, 2020).

La gestión de riesgos se define como el proceso de planificación, ejecución y evaluación de las políticas, estrategias y medidas aplicadas para prever, impedir, reducir, controlar y recuperar los daños ocasionados a la población y el medio ambiente. Comparte en este punto el concepto propuesto por el PNUD, que define a la gestión de riesgos como: "un proceso social que conduce al planeamiento y aplicación de políticas, estrategias, instrumentos y medidas orientadas a impedir, reducir, prever, recuperar y controlar los efectos adversos de fenómenos peligrosos sobre la población, bienes y servicios, así como el ambiente" (PNUD, 2014, p.10).

La participación ciudadana es entendida en este estudio como el proceso a través del cual los ciudadanos comparten las decisiones políticas sobre los asuntos que les afectan, junto con los actores de la gestión pública. Incluye todas las iniciativas de acción colectiva, de reivindicación o de respuesta a las convocatorias que plantea el gobierno para incidir en las decisiones de política pública (Parrés, 2009, Sol Arriaza, 2012 y Díaz Aldret, 2017). De acuerdo con Parrés (2009, p.17), la participación ciudadana parte de la voluntad de incidir del ciudadano que no integra la función pública, en los asuntos públicos de su incumbencia.

La construcción de las teorías de los desastres se ha conformado con los aportes científicos de diversas disciplinas, que contribuyen a la comprensión de los aspectos físicos, sociales, ambientales y sistémicos de los mismos, y han influido en la gestión de riesgos en todo el mundo. La geografía física, los estudios geomorfológicos, la climatología y la hidrología contribuyen a explicar y realizar predicciones de los posibles desastres. Burton, Kates y White clasifican los desastres según su agente causal: climático, geológico, florales y faunísticos. Proponen siete criterios para evaluar el riesgo de los fenómenos ambientales: la magnitud del fenómeno, la frecuencia, la duración, la velocidad en que ocurre desde las primeras señales hasta su máximo, la distribución regular o aleatoria en el tiempo, la extensión territorial que afecta y el grado de concentración espacial en el territorio afectado. (Burton, Kates y White, 1978, Rubiano, 2009).

Durante mucho tiempo las cuestiones sociales y humanas no fueron consideradas en los estudios o la gestión de desastres. Actualmente, el aporte de las Ciencias Sociales permite comprender la problemática social y cultural que acompaña a los desastres, para orientar la gestión, actuación, participación y educación de los ciudadanos. Con estos aportes, el riesgo y el desastre adquieren otra perspectiva, y pasan a ser entendidos como problemas de la adaptación y articulación humana con el ambiente. En este nuevo enfoque, la clasificación de los desastres se amplía a cuatro categorías: naturales, sociales, humanos y seminaturales. Y se clasifican en incontrolables, entre los que se citan a las epidemias, los incendios, los tornados; y los controlables, que incluyen a la inundación, el accidente de tránsito, la polución del agua (Kates, 1970, Rubiano, 2009). Finalmente, Enrico Quarantelli, Russell Dynes, Lavell y otros investigadores de esta problemática, estructuran la "Sociología de los Desastres", dada la necesidad de una mayor precisión en la concepción de 'desastre natural'. Se trata de una concepción multidisciplinaria, 
que incluye los diversos factores y escenarios (Quarantelli, 1985). Sin embargo, la concepción tradicional antropocéntrica todavía es aplicada:

Con los progresos realizados en el desarrollo de conceptos, tecnología y ciencia en las últimas décadas, y la eliminación sistemática de la terminología de 'desastres naturales' de la literatura y las acciones de la Organización para la Reducción del Riesgo de Desastres de las Naciones Unidas (UNDRR por sus siglas en inglés), la noción de que los desastres son 'naturales' sigue siendo generalizada, especialmente entre los políticos, los bancos de desarrollo, las compañías de seguros y el público en general (Lavell \& Lavell, 2020, p.9).

Para Casarrubia (2020), la gestión de riesgo es abordada, frecuentemente, desde un enfoque macro, sin tomar en cuenta la dimensión local y la participación de la población afectada. Es necesario replantear las responsabilidades y compromisos, tanto institucionales como también de los responsables de la planificación del desarrollo, las municipalidades, la gestión ambiental, las comunidades y sus organizaciones, entre otros actores. Para Lavell \& Lavell (2020, p.4):

El riesgo económico asociado con las amenazas físicas, como terremotos e inundaciones, incluye daños directos y pérdidas a elementos construidos y a los activos económicos existentes, seguido de pérdidas futuras vinculadas con el empleo, la producción, el consumo y los ingresos. Estos impactos afectan a diferentes indicadores macro y microeconómicas durante un período prolongado después del evento. El gobierno no tiene ninguna influencia coyuntural sobre el daño directo asociado con tales acontecimientos físicos en el momento en que sucedan. Sin embargo, la planificación, gestión e intervención ex ante influye positiva o negativamente en los niveles potenciales de riesgo, dependiendo de la sensibilidad al riesgo de tales acciones.

Las causales de riesgo y desastre también son cuestionadas por dichos autores, desplazando la visión antropocéntrica de 'desastre natural' a todo lo que causa problemas al funcionamiento de la sociedad humana en su estructura actual, hacia una visión amplia que sitúa al modelo de sociedad y desarrollo como parte del problema. Afirman Lavell \& Lavell (2020, p.5): "Ya sea considerado desde el ángulo de la causalidad de riesgo y los impactos de desastres o desde el ángulo de la recuperación y reconstrucción, el análisis que ofrecemos confirma una visión social de la construcción del riesgo desastre". Al analizar esa visión social, cuestionan la consideración de los elementos naturales como enemigos o amenazas.

La gestión del riesgo a nivel local favorece la conciencia social frente al problema, facilita su vinculación con los proyectos de desarrollo local, y articula los cambios necesarios para obtener mejores resultados (Lavell, 2007, p.5). Concordando con esta posición, Durán Vargas señala que la gestión local representa una "opción de acción directa sobre las condiciones más concretas de inseguridad de las comunidades y que actúa sobre las capacidades y la resiliencia que la historia y la realidad social comunitaria construyen" (Durán Vargas, 2011, p.42).

Estos avances teóricos tuvieron una influencia progresiva en las propuestas y declaraciones internacionales. El Global Assessment Report on Disaster Risk Reduction (GAR) de 2011, presenta el tema del desarrollo y la gestión local, bajo el lema "Revelar el riesgo, replantear el desarrollo". El documento propone un cambio radical en la gestión del riesgo, con compromiso político y responsabilidad en gobiernos centrales y locales, y la participación de la sociedad civil, especialmente las comunidades vulnerables.

El concepto de participación ciudadana, en el marco del Estado Democrático de Derecho (EDD), tiene como antecedente las reflexiones de Robert Dahl (1971), con la propuesta de poliarquía. La descentralización, fortalecida desde los procesos de transición a la democracia en América Latina a finales del siglo XX, impulsa la innovación de propuestas de participación ciudadana en los gobiernos locales. Pero para Jordi Borja $(2000,2002)$, la participación ciudadana sólo es efectiva cuando los poderes locales tienen la atribución de incidir en los poderes que determinan el curso político, cultural, económico y social. Jordi Borja (2000), afirma que la descentralización democrática tiene a la participación ciudadana como su principal estrategia para lograr la efectividad en la gestión local. Para Borja (2002), el ámbito municipal es el lugar ideal de otro tipo de política que implica relaciones próximas y personalizadas entre los electores y los elegidos. 
La innovación de la gestión pública, durante las últimas décadas, se ha vinculado con el diseño de estrategias y mecanismos de participación ciudadana. La experiencia ha demostrado que la participación ciudadana logra una mejor prestación de servicios públicos, en un contexto cada vez más complejo (Díaz Aldret, 2017). Esta tendencia ha generado nuevas formas de participación, en el marco de una nueva propuesta política: la democracia participativa (Franzi, 2009). El desafío de la participación ciudadana, para Sol Arriaza (2012, p.7), en el marco del Estado Democrático de Derecho (EED) fortalece el ejercicio de la ciudadanía y consolida el compromiso social y político del ciudadano. Ricardo Sol Arriaza (2012, p.14) sustenta que la participación ciudadana no se limita a los espacios nacionales, en la era de la globalización. Esta nueva práctica en el ejercicio de la democracia implica un cambio en la cultura política, que incorpore el respeto al otro y la legitimidad de la pluralidad. Pero también señala que los procesos de institucionalización que pueden garantizar la participación ciudadana efectiva son débiles aún, y su aplicación es muy deficiente. La construcción del camino que habrá de seguir el avance de la participación ciudadana en democracia requiere de la formulación de políticas públicas, procedimientos y nuevas prácticas de gestión.

La participación ciudadana se configura como una praxis que incluye el aprendizaje constante y transformador comprometido con la justicia social y la emancipación, en la concepción de Melero (2018). El aprendizaje de las prácticas de participación ciudadana en la gestión de riesgo de desastres sigue ese mismo camino: avanza a partir de las experiencias del propio ejercicio de participación ciudadana en la gestión de riesgo, en un proceso reflexivo y continuo. Lavell \& Lavell (2020, p.4) afirman que: "Al examinar de cerca los diferentes contextos específicos, se puede percibir de qué manera la comprensión de un tipo de desastre ayuda a comprender a otro, y qué aspectos uno puede y debe tener en cuenta al buscar gestionar otros riesgos de desastres".

\section{MATERIALES Y MÉTODOS}

La metodología aplicada fue la revisión bibliográfica sistematizada, desarrollada en sus cuatro fases: búsqueda, evaluación, análisis y síntesis (Codina, 2018). Su función es la identificación de las tendencias y corrientes principales en un área, así como la detección de huecos y oportunidades de investigación. También cumplen la función de demostrar que los autores han examinado de forma rigurosa y sistemática las investigaciones previas, dado el carácter acumulativo de la ciencia. El material bibliográfico revisado comprende: libros, informes de organismos oficiales nacionales e internacionales, legislación nacional y tratados internacionales, artículos y estudios científicos publicados.

El procedimiento de recolección y análisis de datos tuvo un enfoque cualitativo. Los datos obtenidos fueron categorizados en tres dimensiones: histórica, sociodemográfica y ambiental, y política. El estudio se limita a la información concerniente al territorio de la ciudad de Asunción, su área metropolitana y Bajo Chaco. Este territorio es el escenario de las crecidas cíclicas del Río Paraguay que, en sinergia con los fenómenos del Cambio Climático, la urbanización informal y la densificación de la población, han generado un complejo escenario de riesgo urbano creciente.

\section{RESULTADOS}

Los resultados de esta revisión bibliográfica sistematizada fueron analizados en tres dimensiones: dimensión histórica, dimensión sociodemográfica y ambiental, y dimensión política. La dimensión histórica comprende los principales antecedentes y la evolución histórica de la gestión de riesgos de desastres a nivel global, regional, nacional y en el local de este estudio. La dimensión sociodemográfica y ambiental contextualiza la problemática concerniente al riesgo de desastres y su gestión, tomando en cuenta las características geográficas, sociales y ambientales del local de este estudio. En la dimensión política, se presenta una revisión de los avances de las políticas públicas en gestión de riesgo de desastres a nivel internacional, nacional y local.

\section{Dimensión histórica}

La práctica de registrar los desastres, catástrofes y otros fenómenos notables de la naturaleza es muy antigua. Estos registros alertaban a los viajeros y comerciantes sobre los peligros que pudieran encontrar navegando o recorriendo territorios lejanos. Los primeros estudios científicos sobre catástrofes naturales fueron desarrollados en el ámbito de la geografía, a mediados del siglo XVIII. Sus antecedentes remotos se encuentran en las exploraciones territoriales de la época clásica, que definen al desastre a partir del prefijo latino des (sin, carente de), y la palabra griega 
astren (estrella), cuyo significado literal sería "sin estrella". La importancia de la observación de las estrellas, consideradas guías del orden de las cosas en la Tierra por los antiguos, indica que el término resume la idea de situaciones en las que se rompe el orden natural de los fenómenos.

La palabra catástrofe proviene del vocablo griego utilizado en la antigüedad katastrophé, que se compone de los vocablos kata (ruina, desgracia), y strepho, que significa convertir. Literalmente, significa convertir en ruina, de ese modo identificaban los antiguos un cambio brusco que podía alterar el orden, y causar la ruina en un ambiente determinado (Rubiano, 2009, p.243). Pero recién a partir del siglo XVIII se realizan algunas expediciones científicas para recolectar de forma sistemática información territorial, que incluyen datos sobre terremotos, erupciones volcánicas y otros desastres (Rodriguez de la Torre, 1992).

En América del Sur, se puede citar entre los primeros expedicionarios científicos al francés Bouguer, que recorrió Perú, Colombia y Ecuador entre 1739 y 1744. En Paraguay, Félix de Azara inicia en 1782 una expedición a partir de Asunción que dura 20 años, registrando con todo detalle el territorio, los fortines, las comunidades de pobladores y los fenómenos naturales de la región, relato que se publica con el título "Viajes por la América Meridional” (Contreras, 2011).

A partir de 1970, la hipótesis de la influencia de la percepción y acción humana en gran parte de los desastres ha generado muchas investigaciones, especialmente en los países con menor desarrollo. Las situaciones críticas de marginalidad, exclusión y dependencia plantean situaciones de riesgo, vulnerabilidad y dificultades en la recuperación de un desastre. Diversos estudios realizados en Paraguay han demostrado que las comunidades en situación de pobreza habitan, en muchos casos, territorios afectados por inundaciones y contaminación ambiental (Galeano Monti, 2017, Morínigo,1989, Morris, 2014, Rodríguez, 2014, Vuyk 2014, Canese \& Canese, 2015).

\section{Dimensión sociodemográfica y ambiental}

La presente investigación se localiza en la ciudad de Asunción, su área metropolitana y municipios próximos del Bajo Chaco. Según el estudio del componente sociodemográfico desarrollado por Gestión Ambiental (2014), esta zona geográfica se caracteriza por un crecimiento poblacional intenso y desordenado en los últimos años, debido al poder de atracción de la ciudad capital. Mientras tanto, la población de la capital prácticamente no ha crecido durante los últimos años. La población de la ciudad de Asunción, durante el período de este estudio, es de 522.287 habitantes, representando apenas el 7,3 \% de la población del país. El Gran Asunción presenta niveles elevados de pobreza e inequidad urbana, el $17,1 \%$ de la población se encuentra en situación de pobreza. El coeficiente de Gini para las áreas urbanas de Paraguay en 2013 era de 0,4480 , con tendencia descendente. Ese nivel de inequidad se expresa también en un nivel elevado de segregación socioespacial a nivel urbano (GEMA, 2014).

La mitad de las unidades económicas de Paraguay (48,3\%) están ubicadas en Asunción $(18,4 \%)$ y en el Departamento Central $(29,9 \%)$, según datos del Censo Económico Nacional (CEN) de 2011. La tasa de Población Económicamente Activa/Población de 10 y más años de edad en 2014 fue del $65,9 \%$, de acuerdo con la Encuesta Continua de Empleo, en Asunción y áreas urbanas del Departamento Central (GEMA, 2014). La tasa de ocupación en el Área Metropolitana de Asunción, en 2015 , alcanzó al $92,8 \%$ de la población. Sin embargo, el panorama laboral presenta muchas dificultades, entre ellas la subocupación, que alcanza al 15,9\% de la población económicamente activa en el área. El 28,9 \% de los trabajadores asalariados de la región metropolitana de Asunción recibe un salario inferior al mínimo oficial. (Ortiz, Goetz \& Gache, 2020).

El estudio desarrollado por la Iniciativa de Ciudades Emergentes y Sostenibles (ICES) del Banco Interamericano de Desarrollo (2019) señala que el déficit cualitativo habitacional alcanza al $40,5 \%$ de las viviendas en la Región Metropolitana de Asunción. La urbanización informal en esa región ha ido creciendo en las últimas décadas, con asentamientos precarios en zonas periféricas, carentes de infraestructura y servicios. El desarrollo urbano de la región se caracteriza por una gran expansión territorial, periférica y de baja densidad. El mencionado estudio presenta también un análisis prospectivo de crecimiento urbano en esa región. Estima que la población del territorio metropolitano será de 4.300 .000 habitantes en 2030, y 5.600.000 habitantes en 2050. (ICES-BID, 2019). El Plan de Acción elaborado por la Iniciativa de Ciudades Emergentes y Sostenibles (ICES) del Banco Interamericano de Desarrollo (ICES-BID, 2019) identifica las amenazas principales del AMA como de origen natural, generadas por fenómenos hidrológicos y meteorológicos: las inundaciones ocasionadas por crecidas estacionales del río Paraguay, y las inundaciones pluviales 
ocasionadas por los afluentes del río Paraguay. El estudio señala la alteración de la cuenca por efectos del desarrollo urbano no planificado. Históricamente, los registros de inundación por desbordamiento del río Paraguay indican que afecta a más de $1.000 .000 \mathrm{~km} 2$, por ser un río de llanura. La inundación de ribera del AMA y municipios del Bajo Chaco, afecta a 1.650 hectáreas, habitadas por más de 20.000 familias. La amenaza ambiental, proveniente de un proceso natural, se incrementa por las debilidades sociales internas que afectan al territorio de la ciudad de Asunción y municipios circundantes. Las ocupaciones informales del territorio en las riberas de los ríos, cauces de arroyos y humedales de la ciudad de Asunción y su región metropolitana se incrementan, en un proceso de exclusión territorial de las familias con menores ingresos. Si bien se cuenta con un marco regulatorio que establece una franja de dominio público a lo largo de la ribera, cauces de arroyos y humedales, la legislación no evita el uso económico y la ocupación habitacional de las zonas inundables.

La contaminación ambiental es la segunda gran amenaza en el territorio, identificada por ICES-BID (2019) y Sagüi, Estigarribia y Canese (2020) en el territorio. La provisión de los servicios de agua potable, saneamiento, transporte público, vivienda, drenaje pluvial y gestión de residuos sólidos cubre apenas una porción del territorio del Gran Asunción. El servicio de alcantarillado tiene una cobertura del $21 \%$ de las viviendas en la ciudad de Asunción, y el $7,8 \%$ de las viviendas en los demás municipios del Departamento Central. El tratamiento de efluentes cloacales es de apenas $4 \%$, la recolección de residuos sólidos es del $68 \%$, y el sistema de desagüe pluvial cubre el 19\% del territorio en la ciudad de Asunción (ICES-BID, 2019).

La cobertura de recolección de residuos sólidos en el AMA alcanza apenas al $32 \%$ del territorio urbano. A esto se suman los problemas de baja calidad en la recolección y gestión de los residuos. Estas deficiencias tienden a agravar las inundaciones pluviales ocasionadas por lluvias intensas al causar el efecto de obstrucción de las alcantarillas de desagüe pluvial, con el agregado de contaminación por interferencia con las alcantarillas cloacales. El territorio de este estudio no cuenta con sistemas de separación o reducción en origen de residuos sólidos urbanos, salvo algunas experiencias aisladas. La actuación de los segregadores de residuos que trabajan en vertederos y calles de Asunción, y municipios de la zona metropolitana, aporta en alguna medida al reciclado de estos residuos, y brinda un ingreso informal a 6000 personas, entre ellos niños y mujeres embarazadas, pero eleva su exposición al riesgo de contaminación, intoxicación, epidemias e incendios. Lo mismo puede afirmarse de la población que habita en las cercanías de los vertederos de basura a cielo abierto, y de los trabajadores de la zona (ICES-BID, 2019).

Los bañados de Asunción, áreas de inundación en las orillas del río Paraguay, son habitados actualmente por la población de menores ingresos económicos. Además de las inundaciones periódicas que afectan a la zona por la crecida del río, estos territorios se encuentran contaminados por la carencia de infraestructura y el drenaje de los sistemas de alcantarillado sanitario y pluvial de la ciudad, que no cuentan con sistemas de tratamiento de efluentes. La carencia de sistemas de recolección de residuos sólidos en la zona, sumada a la presencia de vertederos clandestinos en el territorio, completa un escenario de riesgos múltiples sanitarios y de degradación ambiental (Sagüi, Estigarribia y Canese, 2020).

\section{Dimensión política}

Desde la visión política internacional, el enfoque del problema de ciudad ha recibido hasta ahora mucho mayor énfasis que el problema de degradación ambiental, vulnerabilidad social, riesgo y desastre urbano como tal (Fernández, 1996, pag. 5), sobre todo por la tendencia generada por parte del Banco Mundial. Por un lado, porque la Gestión Ambiental y Desarrollo Sostenible son vistos, esencialmente, como problemas que atañen a los recursos naturales y al ecosistema. $Y$ por otro, porque de alguna manera, los múltiples componentes de la vulnerabilidad social se han esquivado como componentes explicativos.

El problema de la prevención y mitigación de la degradación ambiental urbana recibió, durante las dos últimas décadas del siglo XX, escaso apoyo por parte de las agencias financiadoras de investigaciones. Los pocos estudios realizados, mantuvieron la mirada hacia el mejoramiento de la capacidad gubernamental y la transferencia de tecnología. En los casos del Banco Mundial, PNUD y el OECD, las soluciones tecnológicas ofrecidas se canalizan a través de gobiernos y se concentran en mecanismos de instrumentación económica y de regulaciones legales, insuficientes para introducir cambios significativos. Los principales problemas identificados, en cuanto a la 
investigación y la gestión ambiental urbana, se refieren a la administración del agua, la prevención y mitigación de desastres, la gestión de desechos sólidos, y la agricultura urbana, dejando de lado la prevención y mitigación de desastres. La falta de una construcción de la totalidad y la derivación de aspectos parciales resultan tan equivocadas como las soluciones tecnocráticas mismas.

Con la publicación, en 1992, del compendio editado por Alcira Kreimer y Mohan Munasinghe sobre "Manejo Ambiental y Vulnerabilidad Urbana" (Kreimer y Munasinghe, 1992), resultado de una conferencia organizada por el Banco Mundial, el tema ambiental urbano se establece en la agenda de discusión internacional, si bien en la literatura académica ya tenía antecedentes. Durante la década de los 80 , el Banco Mundial había sido blanco de creciente crítica por sus políticas "anti ambientales", el fomento de inversiones que modificaban radicalmente el hábitat y el medio ambiente. En el medio urbano, sus preocupaciones habian girado alrededor de las grandes inversiones en infraestructura urbana y los tradicionales "fix" tecnológicos. A pesar de su impacto, Varley (1994) reafirmó el enfoque tecnocrático de la publicación, al concluir que las soluciones planteadas apuntaban esencialmente hacia la transferencia de tecnología, una modernización institucional y una reducción de la vulnerabilidad física de la ciudad. La vulnerabilidad se redujo en gran medida a las características de las edificaciones. El riesgo que enfrenta la población se considera, como dice Varley, un problema de ignorancia o de estar fuera del circuito informacional.

Los argumentos de Varley se encuentran validados, en cierta medida, en una publicación del IDRC de Canadá, editada por Mougeot y Massé, en 1993 (1993, pp. 22-24; traducida al español en Lavell (1994)). Esta publicación, "Gestión Ambiental Urbana: Desarrollando una Agenda Global de Investigación", presenta importantes parámetros para la investigación y acción en el campo de la Gestión Ambiental Urbana. Afirma que las agencias donantes, instituciones y editoriales han sido lentas para llevar a cabo y divulgar investigaciones sobre los problemas ambientales urbanos del Tercer Mundo (Urban Environmental Management Developing a Global Agenda, 1993).

En el siglo XXI, la gestión de riesgos ha pasado de la respuesta ante los desastres, a la reducción de éstos, recalcando el papel esencial que juega la acción humana. En enero del 2005, en Hyogo (Japón) se celebró la novena sesión plenaria de la Conferencia Mundial sobre la Reducción de los Desastres, que aprobó el Marco de Acción 2005-2015, en el que se definen las estrategias para lograr el aumento de la resiliencia de las naciones y las comunidades ante los desastres, impulsando acciones que promuevan la conciencia y el conocimiento que permitan resistir el impacto negativo de los peligros naturales, así como el manejo del riesgo antrópico.

La incorporación de la participación ciudadana en las políticas públicas de gestión de riesgo es todavía más reciente, impulsada por los avances sociocríticos de las teorías de desastres y gestión de riesgos. Las investigaciones recientes sobre la participación ciudadana indican que la misma se incorpora como componente de las políticas públicas para desarrollar gestiones más justas, eficaces y legítimas. El estudio publicado por Díaz Aldret (2017) sobre la participación ciudadana en la gestión y en las políticas públicas, afirma que la participación ciudadana tiene implicancias técnicas y políticas que deben equilibrarse en las prácticas y políticas públicas. En la misma línea, Rosete y Romero (2017) publicaron un estudio sobre la gestión urbana integral, mediante la implementación de mecanismos de inclusión ciudadana y participación en la toma de decisiones.

La investigación sobre los desafíos actuales para la participación ciudadana en la gestión urbana, publicada recientemente por Castro, Gutierrez, Kazak, Szewranski, Kaczmarek \& Wang (2020), identifica los nuevos desafíos para el perfeccionamiento de los procesos de participación ciudadana en la gestión urbana, y los retos que representan para la innovación social. Señala la importancia de mejorar los sistemas de toma de decisiones en la gestión urbana, con participación de la ciudadanía.

La gestión de riesgos, según la Oficina de las Naciones Unidas para la Reducción de Desastres (UNISDR) en su material de Terminología sobre Reducción de Riesgos de Desastres, tiene por objetivo "...evitar, disminuir o transferir los efectos adversos de las amenazas mediante diversas actividades y medidas de prevención, mitigación y preparación (PNUD, 2014). Esta definición aporta una nueva visión de la gestión de riegos, que pasa del enfoque tradicional centrado buscando dar respuesta ante un desastre, a un enfoque preventivo integral, basado en la Gestión y Reducción de Riesgos (GRR). 
En Paraguay, el Parlamento sancionó en 1993 la Ley $N^{\circ}$ 153/93, creando el Comité de Emergencia Nacional (CEN) para dar respuesta a los desastres. En el año 2005, el Congreso Nacional sancionó la Ley №. 2615/05 14, por la cual se crea la Secretaría de Emergencia Nacional (SEN), dependiente de la Presidencia de la República. Su estructura orgánica y funcional se halla establecida en el Decreto N 5.908 del año 2005. Su objetivo primordial es prevenir y contrarrestar los efectos de las emergencias y los desastres, promover, coordinar y orientar las actividades de las instituciones públicas, departamentales, municipales y privadas destinadas a la prevención, mitigación, respuesta, rehabilitación y reconstrucción de las comunidades afectadas por situaciones de emergencia o desastre.

La Política Nacional de Gestión y Reducción de Riesgos establece 5 prioridades:

» Prioridad 1 - Lograr que la reducción del riesgo de desastres sea una prioridad nacional y local con una sólida base institucional para su implementación.

» Prioridad 2 - Conocer el riesgo y tomar medidas.

» Prioridad 3 - Desarrollar una mayor comprensión y concientización.

» Prioridad 4 - Reducir el riesgo.

» Prioridad 5 - Estar preparados y listos para actuar.

No se menciona la participación ciudadana ni sus mecanismos en ninguna de las prioridades. Sin embargo, el tercer pilar estratégico 3: Educación, Comunicación, Participación Ciudadana, aporta algunos puntos a seguir:

» Sistemas educativos formales e informales

» Capacitación técnica

» Sistemas y procedimientos de comunicación interna y externa

» Sistemas y mesas de articulación y dialogo

» Sensibilización y capacitación de actores

» Involucramiento ciudadano

A nivel local, la Municipalidad de Asunción cuenta con una Dirección General De Gestión y Reducción de Riesgos de Desastres (DGRRD). Los demás municipios desarrollan actualmente la conformación de consejos locales de gestión de riesgos, en proceso de implementación.

La gestión de riesgo por desastres en Paraguay es abordada por el Programa de las Naciones Unidas para el Desarrollo (PNUD), con una recopilación de estudios de caso y reflexiones titulada "Gestión y reducción de riesgos, hacia una nueva cultura de prevención en el Paraguay, reflexiones, experiencias y estudios de casos" (PNUD, 2009). Dicha publicación realiza una reflexión sobre el cambio del paradigma internacional relacionado al riesgo, que inició con una mirada centrada particularmente en los desastres, y la creciente preocupación por la gran cantidad de pérdidas que estos producían, tanto en términos de vidas humanas como de recursos e infraestructura económica y social, por el impacto negativo en los ecosistemas frágiles existentes, y por el aumento progresivo en su ocurrencia, severidad e intensidad desde 1960. El documento se enmarca en el cambio de paradigma a nivel internacional, enfocado a la prevención y reducción de desastres, y recalcando el papel esencial que juega la acción humana. Presenta estudios de caso emblemáticos de Municipios que han gestionado situaciones de riesgo de distinta naturaleza, de los que surgen interesantes lecciones aprendidas sobre todo en relación a las acciones de coordinación y complementación de trabajos interinstitucionales para la gestión.

Las publicaciones de investigaciones recientemente desarrolladas sobre la participación ciudadana en el área de este estudio incluyen los estudios publicados en Paraguay durante los últimos años por Morris (2014), Rodríguez (2014), Vuyk (2014), Galeano Monti (2017), Flores López (2020), Canese \& Canese (2016), Canese de Estigarribia, Vuyk Espínola, Sagüi, Ibarra Díaz, Pignata, Velázquez Gauto \& Duré Bañuelos (2019), y Sagüi, Estigarribia y Canese (2020). Flores López (2020) analiza la situación de riesgo en asentamientos precarios en bordes de arroyos, y la tecnología de gestión del hábitat en Luque, Paraguay. En la región del Chaco, el estudio desarrollado por Estigarribia \& Sagüi (2020) presenta los resultados de una investigación cualitativa sobre la capacidad de respuesta ante riesgos y desastres de las comunidades indígenas de esta región. 
La investigación de Néstor Sagüi, Estigarribia y Canese (2020) analiza los riesgos actuales y derechos socio-ambientales en urbanizaciones populares del Área Metropolitana de Asunción. El estudio de los procesos de urbanización popular en el Gran Asunción, desarrollada por Canese de Estigarribia, Vuyk Espínola, Sagüi, Ibarra Díaz, Pignata, Velázquez Gauto \& Duré Bañuelos (2019), aporta un mapeo de los procesos de asentamiento, organización y movilización, en zonas vulnerables a las inundaciones. El libro publicado por Canese \& Canese (2016) describe la conveniencia de la defensa costera para los bañados de Asunción, que, según los autores mencionados, podría terminar con el riesgo permanente de las inundaciones en dicha zona. Es el resultado de un estudio realizado con la Coordinadora General de Organizaciones Sociales y Comunitarias de los Bañados de Asunción (COBAÑADOS).

Sobre la temática de la participación ciudadana en Asunción y su área de influencia, el libro publicado por Flores, Ibarra, Prieto, Estigarribia, \& Vuyk (2018) aporta lineamientos para la gestión urbana participativa en la ciudad de Asunción. El estudio sobre liderazgo, participación y construcción de ciudadanía en urbanizaciones populares de Asunción (Canese de Estigarribia, 2019) presenta los resultados de un estudio cualitativo sobre liderazgo social y participación ciudadana en urbanizaciones populares de Asunción, Paraguay. Estas comunidades excluidas de la ciudad formal han desarrollado procesos innovadores de participación ciudadana, para hacer frente a los desastres y reclamar sus derechos. Los resultados obtenidos en el estudio indican la emergencia del liderazgo participativo y solidario, que busca reducir las exclusiones, promover la solidaridad y promover nuevos mecanismos de participación en la gestión de riesgo ante las inundaciones que afectan a sus comunidades.

\section{DISCUSIÓN}

Los resultados de esta revisión sistematizada de literatura permiten iniciar un análisis crítico de las dimensiones del problema de la implementación de la participación ciudadana en la gestión de riesgo en Asunción y su zona de influencia. En la dimensión histórica, los datos obtenidos indican que la participación ciudadana se limita a la movilización autónoma de las organizaciones comunitarias de base, con escaso poder de decisión, incidencia y control en la gestión de riesgos a nivel nacional y local. La dimensión sociodemográfica y ambiental revela la situación de vulnerabilidad y amenazas presentes en Asunción y su región metropolitana.

Los resultados de la revisión de los avances en la política internacional y su influencia a nivel local, mencionan la participación ciudadana en la gestión de riesgos de desastres, pero no establecen los mecanismos apropiados, vinculantes, para su implementación efectiva. Las prácticas de gestión política a nivel nacional y local dejan la participación ciudadana en segundo plano, ante la urgencia de las medidas paliativas que aplican en situación de desastre. Los fondos y adquisiciones aceleradas evitan todo tipo de incidencia y control que pudieran ejercer los ciudadanos, ante las prácticas tradicionales de corrupción y prebendarismo.

\section{CONCLUSIONES}

La discusión de los resultados obtenidos en la presente investigación identifica las dimensiones y desafíos que enfrenta el proceso de implementación de la participación ciudadana en la gestión de riesgo en la ciudad de Asunción, Paraguay, y municipios que la circundan. El paradigma emergente en la formulación teórica de la gestión de riesgo de desastres revela un gran cambio y un nuevo camino a seguir, que obligan a repensar las estrategias y mecanismos a ser implementados. El propósito de la gestión de riesgo de desastres ha pasado de brindar una respuesta reactiva y paliativa a los desastres ya ocurridos, a la prevención o reducción de éstos, recalcando el papel esencial que juega la acción humana. Las fuentes consultadas en este estudio mencionan la importancia estratégica de la participación ciudadana en la gestión de riesgo de desastres, tanto en las declaraciones internacionales vigentes, como en los documentos nacionales que delinean la política pública del país en gestión de riesgo de desastres. Sin embargo, la revisión de informes de investigación y artículos científicos publicados cuestionan la implementación de la participación ciudadana de forma integral, señalando que en la práctica se limita al aspecto formal, con escaso poder de la ciudadanía en la incidencia y el control de la gestión de riesgo.

La situación de exclusión social, informalidad y precariedad habitacional de los sectores vulnerables de la población urbana en la ciudad de Asunción y su región metropolitana, se agrava ante la escasa participación ciudadana efectiva en las comunidades de alta vulnerabilidad y mayor 
riesgo en el territorio. La gestión de riesgo de las instituciones gubernamentales nacionales y locales, en ese contexto, es muy limitada y se restringe a brindar una respuesta reactiva, muy poco efectiva y sin participación de los ciudadanos afectados o de la sociedad civil en general.

El análisis de la dimensión política identifica la persistencia de barreras históricas, en contraste con la emergencia nuevos caminos esperanzadores en el proceso de implementación de las políticas públicas de gestión de riesgo establecidas recientemente en el país, que incluyen a la participación ciudadana como una prioridad estratégica. Sin embargo, en las instancias operativas, la confrontación con las prácticas políticas tradicionales impide la generación de nuevos mecanismos que garanticen la participación ciudadana desde la toma de decisiones, la planificación, la ejecución y el monitoreo de la gestión.

La partidización de las organizaciones populares funcionales a los grupos de poder sigue siendo la estrategia de legitimación del uso prebendario de los fondos y recursos disponibles para gestión de riesgo. La continuidad de estas prácticas, como mecanismo de dominación política desde el poder, siguen siendo habituales, a pesar de ser incompatibles con los avances propuestos en las políticas públicas establecidas en los últimos años. Estas prácticas de corrupción y prebendarismo de la ayuda de emergencias, a nivel nacional y municipal, deberán ser erradicadas para dar lugar a la implementación de la nueva visión de gestión de riesgo por desastres plasmada en las declaraciones internacionales y políticas públicas nacionales, que establecen un nuevo estilo de gestión participativa.

El principal desafío a vencer para la implementación efectiva de la participación ciudadana en la gestión de riesgo de desastres se encuentra en la reconstrucción de la cultura política ciudadana, tomando como base fundamental la nueva visión y los cambios anunciados en los encuentros internacionales y políticas públicas de gestión de riesgo a nivel nacional. Para lograrlo, es necesario formar una nueva cultura política basada en la participación ciudadana para la gestión de riesgo de desastres. Sin embargo, en el marco de esta contradicción crece la gestión solidaria y la movilización de las organizaciones comunitarias, en demanda de sus derechos sociales y políticos. La emergencia de liderazgos participativos y la conformación de organizaciones comunitarias autónomas en algunos municipios y zonas de mayor vulnerabilidad, mejora la viabilidad de la participación ciudadana en la gestión de riesgo de desastres en sus territorios.

Las limitaciones de este estudio derivan de su opción metodológica, basada en una revisión bibliográfica sistematizada de alcance exploratorio, y las limitaciones propias de los procesos de selección y evaluación de las fuentes de datos. Por tanto, los aportes de este estudio no son conclusivos, sino más bien referencias para la continuidad de esta línea de investigación en el contexto urbano del Gran Asunción. Con esas limitaciones, a partir de los aportes emergentes en este estudio se realizan algunas recomendaciones:

» Conformar estructuras especializadas en gestión de riesgo de desastres en cada una de las municipalidades del Gran Asunción, con participación directa de las organizaciones de las comunidades.

» Generar una base de datos a nivel municipal, fiable, pública y transparente, que incluya los riesgos de desastres, la vulnerabilidad, la capacidad, el grado de exposición de la población afectada, las características de las amenazas y la posible secuencia de efectos, las afectaciones y las pérdidas.

» Incorporar los protocolos de actuación en prevención, evasión, mitigación y superación de riesgos de desastres, con participación directa de las organizaciones de las comunidades afectadas.

» Fomentar la investigación científica en materia de gestión de riesgo de desastres en los municipios del Gran Asunción.

» Capacitar en gestión de riesgo, de forma sistemática y continua, a los gestores municipales, técnicos y líderes de las organizaciones comunitarias.

» Implementar programas de formación específica en participación ciudadana en la gestión de riesgo de desastres, dirigidos a las organizaciones comunitarias de la población que habita en los territorios con mayor riesgo. 


\section{AGRADECIMIENTOS}

Este estudio forma parte del PROYECTO CONACYT-PROCIENCIA: LECCIONES APRENDIDAS DESDE LA PARTICIPACIÓN CIUDADANA EN LA GESTIÓN DE RIESGOS EN ASUNCIÓN, ÁREA METROPOLITANA Y BAJO CHACO, en desarrollo por la organización Cultura y Participación y la Escuela de Ciencias Sociales y Políticas, Universidad Nacional de Asunción, Asunción, Paraguay.

\section{REFERENCIAS}

Borja, J. (2000). Descentralización y participación ciudadana. CESEM.

Borja, J. (2002). Gobiernos locales, políticas públicas y participación ciudadana. Aportes. Revista de la Asociación de Administradores Gubernamentales, 9(19), 57-65.

Canese de Estigarribia, M. I. C. (2019). Liderazgo, participación y construcción de ciudadanía en urbanizaciones populares de Asunción, Paraguay. Acta Scientiarum. Human and Social Sciences, 41(2), e46208-e46208.

Canese de Estigarribia, M. I., Vuyk Espínola, C. M., Sagüi, N. J., Ibarra Díaz, G. A., Pignata, R. M., Velázquez Gauto, N. A., Godoy, P.; Allende J., Villalba, G. \& Duré Bañuelos, V. (2019). Urbanización popular en la ciudad de Asunción, Paraguay. Revista INVI, 34(95), 9-42.

Canese, M.I. (2007). Sociedad y cultura urbana: convicencia ciudadana en plazas y parques de Asunción. Revista Internacional de Investigación en Ciencias Sociales, 3(2), 127-142.

Canese, R., \& Canese, M. (2016). La lucha por la tierra en Asunción: la conveniencia de la defensa costera para los bañados. Cobañados, Coordinadora General de Organizaciones Sociales y Comunitarias de los Bañados de Asunción.

Casarrubia, J.D.R. (2020). Indicadores de vulnerabilidad social como herramienta de planificación y gestión de riesgos ambientales en Colombia. Vestigium Ire, 14(1), 41-55.

Castro, D. G., Gutierrez, V. D. E., Kazak, J., Szewranski, S., Kaczmarek, I., \& Wang, T. (202o). Nuevos desafíos para el perfeccionamiento de los procesos de participación ciudadana en la gestión urbana. Retos para la innovación social. Cuadernos de Gestión, 2o(1), 41-64.

Contreras Roqué, J. R. (2011) Félix de Azara. Su vida y su época. Tomo Segundo. El despertar de un naturalista: la etapa paraguaya y rioplatense (1782-1801). Diputación Provincial de Huesca, Zaragoza.

Dahl, R. (1971). La poliarquía. Participación y oposición. Tecnos.

Díaz Aldret, A. (2017). Participación ciudadana en la gestión y en las políticas públicas. Gestión y política pública, 26(2), 341-379.

Franzi, M.P. (2009). Participación y calidad democrática: evaluando las nuevas formas de democracia participativa. Ariel.

Escobar de Morel, M. (2012). La participación ciudadana en Paraguay. Análisis a partir de la transición democrática. Revista Internacional de Investigación en Ciencias Sociales, 8(1), 119-140.

Estigarribia, S.E \& Sagüi, N.J. (2020). Plan indígena de acción climática y reducción del riesgo de desastres. En: Proyecto Fortaleciendo capacidades para el desarrollo de iniciativas de protección y conservación de los recursos bioculturales de los territorios indígenas, desde la gestión integral y la promoción de medidas proactivas frente al cambio climático. FAPI-WWF-TEBTEBBA-Tamal Pais Trust.

Fernandez, M.A. (Compiladora). (1996). Ciudades en riesgo degradación ambiental, riesgos urbanos y desastres. La red, Red de Estudios Sociales en Prevención de Desastres en América Latina.

Flores López, A.R. (2020). Estudios de situaciones de riesgo en Asentamientos Precarios en bordes de Arroyos, como Tecnología de gestión del hábitat. Caso: Luque, Paraguay. Arquitecno, (15), 17-26.

Flores, A. R., Ibarra, G., Prieto, V., Estigarribia, S., \& Vuyk, C. (2018). Gestión urbana y participación en la ciudad de Asunción. Cultura y Participación para el Cambio Social.

Freire, P. (1985). The politics of education: Culture, power, and liberation. Greenwood Publishing Group.

Galeano Monti, J. (2017). Territorios de exclusión social. Enfoque territorial.

Giroux, H.A. (2010). Rethinking education as the practice of freedom: Paulo Freire and the promise of critical pedagogy. Policy Futures in Education, 8(6), 715-721.

Lavell, A. \& Lavell, C. (2020). El COVID-19: Relaciones con el riesgo de desastres, su concepto y gestión. Red de Estudios Sociales en Prevención de Desastres en América Latina. https://www.desenredando. org/public/2020/Lavell_2020-07_Covid-19_y_Desastre_Final.pdf. 
Mansilla, E. (2010). Riesgo Urbano y Políticas Públicas en América Latina: La Irregularidad y el Acceso al Suelo. En Global Assessment Report on Disaster Risk Reduction. UNISDR.

Melero, H. (2018). Espacios y prácticas de participación ciudadana. Análisis y propuestas educativas desde un enfoque intercultural. Sociedad Iberoamericana de Pedagogía Social. https:/gredos.usal.es/ handle/10366/140033

Morínigo, J.N. (1989). Pobreza Urbana y Vivienda. CIPAE.

Morris, F. (2014). Diagnóstico del Sector Vivienda. Banco Interamericano de Desarrollo.

Mougeot, L. (1993). "El Programa de Gestión Ambiental Urbano”. En Lavell A. (comp), 1994. La Red.

Parés, M. \& Resende, P. (2009). Tipos de prácticas y experiencias de participación promovidas por la administración pública: Sus límites y radicalidades. En Marc Parés (coord.), Participación y calidad democrática: Evaluando las nuevas formas de democracia participativa. Ariel \& Generalitat de Catalunya, pp. 77-10o.

Proyecto Naciones Unidas para el Desarrollo PNUD. (2009). Gestión y Reducción de Riesgos, Hacia Una Nueva Cultura de Prevención en el Paraguay, Reflexiones, experiencias y estudios de casos. PNUD.

Proyecto Naciones Unidas para el Desarrollo PNUD (2014). Gestión del Riesgo de Desastres. Centro Regional del PNUD para América Latina. PNUD. https://www.undp.org/content/dam/ rblac/docs/Research\%2oand\%2oPublications/Crisis\%2oPrevention\%2oand\%2oRecovery/ Brochure_Desastres_sp.pdf

Quarantelli, E. (1985). ¿Qué es el desastre? La necesidad de aclaración en la definición y conceptualización en la investigación. Centro de Investigación de Desastres de la Universidad de Delaware.

Ramírez Cortés, F. (2015). La Gestión del Riesgo Urbano en América Latina Recopilación de Artículos. EIRD. https://www.eird.org/plataforma-tematica-riesgo-urbano/recopilacion-de-articulos/lagestion-del-riesgo-urbano-en-america-latina.pdf

Rodríguez de la Torre, F. (1992). La geografía y la historia de los sismos. Geocrítica, (97), 9-69. http:// www.ub.edu/geocrit/geog7.htm

Rodríguez, J.C. (2014) Vínculos y territorios. Actores y capital social en el Departamento Central del Paraguay. Ed. ID. \& Gobernación del Departamento Central Central.

Rosete, N.L.R., \& Romero, M.L.G. (2018). Gestión Urbana Integral. Revista Planeo, 67(1), 1-10. http:// revistaplaneo.cl/wp-content/uploads/Arti\%CC\%81culo_Rami\%CC\%81rez.pdf

Rubiano, M.T.M. (2009). Los geógrafos y la teoría de riesgos y desastres ambientales. Perspectiva Geográfica: Revista del Programa de Estudios de Posgrado en Geografía, (14), 241-263.

Sagüi, N.J., de Estigarribia, M.I.C., \& Canese, S.E.E. (2020). Derechos socio-ambientales en urbanizaciones populares del Área Metropolitana de Asunción, Paraguay. Revista F@ ro, 1(31).

Urban Environmental Management Developing a Global Agenda. (1993). IDCR, 2 volúmenes. Ottawa.

Vuyk, C. (2014). Ribera del río, desarrollo de la ciudad: proyectos en disputa. Plataforma Temática de Riesgo Urbano. UNISDR, Las Américas, \& CEPAG.

Zemelman, H. (2011). Los horizontes de la razón, Tomo III. Anthropos, Universidad de Caldas.

Zemelman, H. (2005). Voluntad de conocer: el sujeto y su pensamiento en el paradigma crítico (Vol. 47). Anthropos Editorial.

Zemelman, H. (1989). De la historia a la política; la experiencia de América Latina. Siglo XXI \& México Biblioteca América Latina: Actualidad y Perspectivas. 